\title{
Acknowledgement to Reviewers of Plants in 2018
}

\section{Plants Editorial Office}

MDPI, St. Alban-Anlage 66, 4052 Basel, Switzerland

Published: 9 January 2019

Rigorous peer-review is the corner-stone of high-quality academic publishing. The editorial team greatly appreciates the reviewers who contributed their knowledge and expertise to the journal's editorial process over the past 12 months. In 2018, a total of 114 papers were published in the journal, with a median time to first decision of 15.4 days and a median time to publication of 37 days. The editors would like to express their sincere gratitude to the following reviewers for their cooperation and dedication in 2018:

Acosta, Ivan F.

Adamakis, Ioannis-Dimosthenis

Agnieszka, Szopa

Agudelo-Romero, Patricia

Alba, Juan

Aleksandrowicz-Trzcińska, Marta

Alexopoulos, Athanassios

Alhousari, Fadi

Alizadeh, Hossein

Alqudah, Ahmed

Altemimi, Ammar

Álvarez-Fernández, M. Antonia

Ambrose, Chris

Amirkhani, Masoume

Ancuceanu, Robert

Andreev, Konstantin

Anwar, Rokebul

Ates, Serkan

Bahmani, Ramin

Bai, Hua

Bajgain, Rajen

Baldrich, Patricia

Ballizany, Wouter

Banilas, Georgios

Barba, Francisco J.

Barchenger, Derek

Barman, Apurba

Barriocanal, Carles

Barta, Csengele
Bazos, Ioannis

Becker, Jörg

Benvenuto, Eugenio

Bhattacharjee, Sonali

Bhowmik, Arnab

Bleckmann, Andrea

Blein, Thomas

Bogdan, Ileana

Bones, Atle

Bonga, Jan Max

Boss, Paul

Brückner, Adrian

Brunetti, Cecilia

Bubici, Giovanni

Bunce, James

Burghardt, Liana T.

Cabot, Catalina

Caldo, Kristian Mark

Campbell, Michael

Canini, Antonella

Cao, Zhe

Capasso, Raffaele

Cardinale, Massimiliano

Cardoso, Susana

Carrasco, Pedro

Carrubba, Alessandra

Carvajal, Micaela

Caser, Matteo

Cazzato, Eugenio 
Celiński, Konrad

Champion, Cody

Chang, Hao-Xun

Chang, Young Ki

Chen, Dijun

Chen, Haotong

Chen, Zhonghua

Cheng, Juei-Tang

Chin, Young-Won

Chizzola, Remigius

Çiçek, Serhat Sezai

Ciulu, Marco

Commisso, Mauro

Constantinidis, Theophanis

Contreras, María Del Mar

Costa, Alex

Crain, Jared

Croce, Anna Cleta

Cullis, Christopher

Dąbrowska, Jolanta

Daras, Gerasimos

Davies, Julia

De Bellis, Luigi

De Diego, Nuria

De Mieri, Maria

De Tullio, M.C.

Del Giudice, Rita

Del Río Celestino, Mercedes

Deshmukh, Rupesh

Devireddy, Amith

Dey, Prabuddha

Dinu-Pîrvu, Cristina Elena

Dong, Yibo

Donno, Dario

Eichberg, Carsten

Elbasyoni, Ibrahim

Elbaum, Rivka

Eleftheriou, Eleftherios P.

Endresen, Dag

Engelberth, Jurgen

Fagnano, Massimo

Falistocco, Egizia

Foereid, Bente
Frew, Adam

Frugis, Giovanna

Galindo-González, Leonardo

Gallemí, Marçal

Gangola, Manu Pratap

Gao, Xiaolong

Garrido-Cardenas, Jose Antonio

Ge, Yufeng

Gervasi, Teresa

Ghani Zadeh, Hossein

Gianinetti, Alberto

Giannakoula, Anastasia

Giovannini, Annalisa

Gladish, Daniel K.

Gniewosz, Małgorzata

Gombojav, Ariunbold

Grkovic, Tanja

Guido, Luis F.

Gujas, Bojan

Gulati, Vandana

Guy, Charles L.

Hackauf, Bernd

Hammerschmidt, Ray

Hammes, Ulrich

Hano, Christophe

Harris, Philip J.

Hashiguchi, Akiko

Hatano, Tsutomu

Hay, Angela

Heaney, Liam

Heilmann, Mareike

Heiskanen, Juha

Helaly, Soleiman

Herrero, Baudilio

Hildreth, Sherry

Himeno, Hyouta

Hiruma, Kei

Ho, Honhing

Hoch, Günter

Holalu, Srinidhi

Hooykaas, Paul J.J.

Horgan, Finbarr G.

Horim, Lee 


\author{
Hu, Guanggan \\ $\mathrm{Hu}$, Zhenbin \\ Huang, Lei \\ Huggins, Trevis \\ Ischebeck, Till \\ Islam, M. Nurul \\ Iwasaki, Naoto \\ Janga, Madhusudhana \\ Jansen, Marcus \\ Jaradat, Nidal \\ Jarquin, Diego \\ Jarret, Robert L. \\ Jiang, Shu-Ye \\ Johnson, Kim \\ Jones, Huw \\ Jordão, António Manuel \\ Juliano, Claudia \\ Kaiser, Elias \\ Kanaoka, Masahiro \\ Katsuhara, Maki \\ Keplinger, Tobias \\ Khallouki, Farid \\ Kim, Ki Hyun \\ Kimbaris, Athanassios S. \\ Kirst, Henning \\ Knox, Paul \\ Kobayashi, Natsuko I. \\ Kolukisaoglu, Üner \\ Kondo, Yuki \\ Kopecký, David \\ Kosova, Klara \\ Kozaki, Akiko \\ Kozlov, Mikhail \\ Kumar, Manu \\ Kumpf, Robert \\ Kuo, Ping-Chung \\ Kurczynska, Ewa \\ Lachman, Jaromir \\ Lahiri, Sriyanka \\ Lang, Ingeborg \\ Larena, Inmaculada \\ Latosińska, Jolanta \\ Lee, Sang-Han
}

Leiva-Brondo, Miguel

Lenhard, Michael

Lewis, James D.

Li, Mao

Li, Wanlong

Li, Wenyi

Li, Xianran

Liao, Pei-Chun

Libault, Marc

Lidon, Fernando

Liolios, Konstantinos A.

Lionetti, Vincenzo

Liu, Yongliang

Long, Yuchen

Longo, Rocco

$\mathrm{Lu}$, You

Lucarini, Massimo

Lukinavicius, Grazvydas

Lupini, Antonio

Lütken, Henrik

Ma, Guojia

Macnack, Natasha

Malcolm, Brendon

Manfredini, Stefano

Manjunath, Manubolu

Marla, Sandeep

Martinez, Manuel

Martínez, Sidonia

Martins, Natália

Martos, Soledad

Maruyama-Nakashita, Akiko

Mastrangelo, Anna M.

Matthes, Annemarie

Mattoo, Autar

Mavian, Carla

Mccray, Mabry

McCubbin, Andrew

Menzel, Christopher M.

Miguel, Maria

Milella, Luigi

Minocha, Subhash C.

Mishra, Sasmita

Miyamoto, Koji 


\author{
Morello, Laura \\ Mori, Kazuhiro \\ Murai, Koji \\ Murata, Jun \\ Murata, Toshihiro \\ Murray, Jeremy \\ Nachimuthu, Gunasekhar \\ Nadia, Bazihizina \\ Nakka, Sridevi \\ Nandi, Saikat \\ Neri, Davide \\ Notaguchi, Michitaka \\ Nowicki, Marcin \\ Omar, Ahmad \\ Orfila, Caroline \\ Pagnotta, Mario A. \\ Pandolfini, Tiziana \\ Panteris, Emmanuel \\ Pappas, Maria L. \\ Paul, Matthew \\ Peirats-Llobet, Marta \\ Pentzold, Stefan \\ Pereira, Ana Luisa \\ Perrine-Walker, Francine \\ Petrakis, Panos. V. \\ Pettolino, Filomena \\ Pezzotti, Mario \\ Pidatala, Venkata Ramana \\ Pollastrini, Martina \\ Proestos, Charalampos \\ Qaderi, Mirwais \\ Qualset, Calvin O. \\ Rai, Dilip \\ Ramegowda, Venkategowda \\ Ranjit, Suman \\ Rascio, Agata \\ Rayon, Catherine \\ Reed, Kevin F.M. \\ Ren, Hong \\ Ren, Yulin \\ Renault, Sylvie \\ Renna, Massimiliano \\ Ricci, Antonella
}

Riechmann, José Luis

Rodrigo Comino, Jesús

Rodríguez-Seijo, Andres

Roh, Changhyun

Roscoe, Thomas

Rozhon, Wilfried

Rubio Valverde, Lourdes

Ruelland, Eric

Sabater, Bartolome

Sabella, Erika

Saito, Yoshiro

Salas, José Blanco

Santino, Angelo

Sarkar, Saswata Sankar

Sato-Nara, Kumi

Schäffner, Anton

Schaller, Jörg

Scherf, Katharina A.

Schläppi, Michael R.

Schwitzguebel, Jean-Paul

Scognamiglio, Monica

Serafini-Fracasini, Danatella

Setzer, William N.

Shafian, Sanaz

Shen, Jianqiang

Sheng, Ruilong

Shimoda, Hiroshi

Shrestha, Krishna

Shunmugam, Arun

Silar, Philippe

Singh, Raksha

Siracusa, Laura

Smith, Mark A

Sobeh, Mansour

Somssich, Marc

Song, Guoqing

Sorieul, Mathias

Spiegler, Verena

Springer, Patricia

Stadler, Marc

Strati, Irini F.

Suleria, Hafiz

Sweeney, Jon 
Symkal, Petr

Syrpas, Michail

Takeshi, Miki

Taranto, Francesca

Tattini, Massimiliano

Tedesco, Idolo

Tellez, Trinidad Ruíz

Terzaghi, William

Thammina, Chandra

Thapa, Sushil

Thijs, Sofie

Thilmony, Roger

Thiruvengadam, Muthu

Thomas, Elizabeth

Tomi, Félix

Torres-Martínez, Lorena

Torricelli, Renzo

Tripodi, Pasquale

Tsai, Mei-Lin

Tsopmo, Apollinaire

Turgeon, Robert

Tytła, Malwina

Umehara, Mikihisa

Underwood, William

Upadhyay, Rakesh K.

Uygun, Sahra

Van Esse, Wilma

Van Heusden, Adriaan W

Vaughan, Martha M
Vaz, Josiana

Venäläinen, Salla

Venturi, Francesca

Verger, Stéphane

Vicente, Claudia

Volaire, Florence

Walker, Berkley James

Wang, Kun

Wardill, Hannah R.

Wege, Stefanie

Wehling, Peter

Wenkel, Stephan

Wientjes, Emilie

Wiggins, Greg

Wilkinson, Mark D.

$\mathrm{Wu}$, Keqiang

$\mathrm{Wu}$, Qingyu

Xiao, Jun

$\mathrm{Xu}$, Peng

Yang, Inho

Yordanov, Yordan

Yoshioka, Keiko

Zambrano-Wilson, Maria Clemencia

Zamyatnin, Jr., Andrey A.

Zarra, Ignacio

Zavafer, Alonso

Zdunek, Artur

Zhou, Bangjun

Zuo, Ran

(C) 2019 by the author. Licensee MDPI, Basel, Switzerland. This article is an open access article distributed under the terms and conditions of the Creative Commons Attribution (CC BY) license (http://creativecommons.org/licenses/by/4.0/). 